\title{
Fluorouracil sensitivity in a head and neck squamous cell carcinoma with a somatic DPYD structural variant
}

\author{
Elisa Majounie, ${ }^{1}$ Kathleen Wee, ${ }^{1}$ Laura M. Williamson, ${ }^{1}$ Martin R. Jones, ${ }^{1}$ \\ Erin Pleasance, ${ }^{1}$ Howard J. Lim, ${ }^{2}$ Cheryl Ho, ${ }^{2}$ Daniel J. Renouf ${ }^{2,3}$ Stephen Yip, ${ }^{4}$ \\ Steven J.M. Jones, ${ }^{1,5,6}$ Marco A. Marra, ${ }^{1,6}$ and Janessa Laskin ${ }^{2}$ \\ ${ }^{1}$ Canada's Michael Smith Genome Sciences Centre, Vancouver, British Columbia V5Z 4S6, Canada; \\ ${ }^{2}$ Department of Medical Oncology, BC Cancer, Vancouver, British Columbia V5Z 4E6, Canada; ${ }^{3}$ Pancreas \\ Centre BC, Vancouver, British Columbia V5Z 1L8, Canada; ${ }^{4}$ Department of Pathology and Laboratory \\ Medicine, University of British Columbia, Vancouver, British Columbia V6T 2B5, Canada; ${ }^{5}$ Department of \\ Molecular Biology and Biochemistry, Simon Fraser University, Vancouver, British Columbia V5A 1S6, Canada; \\ ${ }^{6}$ Department of Medical Genetics, University of British Columbia, Vancouver, British Columbia V6H 3N1, Canada
}

Corresponding author: jlaskin@bccancer.bc.ca

(C) 2020 Majounie et al. This article is distributed under the terms of the Creative Commons Attribution-NonCommercial License, which permits reuse and redistribution, except for commercial purposes, provided that the original author and source are credited.

Ontology term: squamous cell carcinoma of the skin

Published by Cold Spring Harbor Laboratory Press

doi:10.1101/mcs.a004713
Abstract Head and neck squamous cell carcinoma (HNSCC) is one of the most common cancers worldwide and represents a heterogeneous group of tumors, the majority of which are treated with a combination of surgery, radiation, and chemotherapy. Fluoropyrimidine (5-FU) and its oral prodrug, capecitabine, are commonly prescribed treatments for several solid tumor types including HNSCC. 5-FU-associated toxicity is observed in $~ 30 \%$ of treated patients and is largely caused by germline polymorphisms in DPYD, which encodes dihydropyrimidine dehydrogenase, a key enzyme of 5-FU catabolism and deactivation. Although the association of germline DPYD alterations with toxicity is well-described, the potential contribution of somatic DPYD alterations to 5-FU sensitivity has not been explored. In a patient with metastatic HNSCC, in-depth genomic and transcriptomic integrative analysis on a biopsy from a metastatic neck lesion revealed alterations in genes that are associated with 5-FU uptake and metabolism. These included a novel somatic structural variant resulting in a partial deletion affecting $D P Y D$, a variant of unknown significance affecting SLC29A1, and homozygous deletion of MTAP. There was no evidence of deleterious germline polymorphisms that have been associated with 5-FU toxicity, indicating a potential vulnerability of the tumor to 5-FU therapy. The discovery of the novel DPYD variant led to the initiation of 5-FU treatment that resulted in a rapid response lasting $17 \mathrm{wk}$, with subsequent relapse due to unknown resistance mechanisms. This suggests that somatic alterations present in this tumor may serve as markers for tumor sensitivity to 5-FU, aiding in the selection of personalized treatment strategies.

[Supplemental material is available for this article.]

\section{INTRODUCTION}

Head and neck squamous cell carcinoma (HNSCC) is one of the most common cancers worldwide and represents a heterogeneous group of tumors originating from the squamous epithelium of the oral cavity, oropharynx, larynx, and hypopharynx. Human papillomavirus (HPV) infection is associated with $60 \%-70 \%$ of head and neck cancers. HPV-negative HNSCC tumors, however, tend to have a worse prognosis and response to treatment compared with HPV-positive tumors (Berman and Schiller 2017; Fung et al. 2017). Irrespective of 
HPV status, the majority of HNSCC patients are treated with a combination of surgery, radiation, and chemotherapy (Adelstein et al. 2017). Fluoropyrimidine (5-FU) and its prodrug capecitabine are a frequently prescribed systemic therapy in the treatment of several solid tumor types including HNSCC (Diasio and Harris 1989). Importantly, 5-FU-related toxicity is observed in $30 \%$ of treated patients (Meulendijks et al. 2015). Dihydropyrimidine dehydrogenase (DPD) is a rate-limiting enzyme of 5-FU catabolism and deactivation (Diasio and Harris 1989). Consequently, DPD activity moderates response to 5-FU, and DPD deficiency as a result of germline polymorphism is considered a major cause of 5-FU-associated toxicity (van Kuilenburg 2004). Deleterious variants in DPYD, the large gene encoding DPD, have been described as significantly impacting enzymatic activity (Etienne-Grimaldi et al. 2017; van Kuilenburg et al. 2017; Henricks et al. 2018).

To date, few studies have fully characterized the somatic or germline genomic landscape of DPYD in cancer patients (Etienne-Grimaldi et al. 2017). Here, we present the case study of a patient with HNSCC who had a biopsy that underwent in-depth genomic and transcriptomic integrative analysis. We identified a number of alterations that are associated with 5-FU uptake and metabolism, including a novel somatic structural variant resulting in a partial deletion affecting the DPYD gene as well as a homozygous variant of unknown significance affecting SLC29A1 and homozygous deletion of MTAP. There was no evidence of deleterious germline polymorphisms that have been associated with 5-FU toxicity. Given the welldescribed toxicity to 5-FU associated with deleterious germline variants affecting DPYD, we hypothesized that the somatic DPYD structural variant may have rendered the tumor to be sensitive to 5-FU and report the subsequent response to treatment.

\section{RESULTS}

\section{Clinical Presentation}

The patient was a 69-yr-old gentleman of East-Asian extraction who presented with a 4 mo history of oral discomfort and was found to have an ulcer on his right maxillary palate. The patient was a lifelong never-smoker, drank alcohol approximately once per month, and had no other significant medical history. Pathology evaluation of the resected ulcer (with a right hemimaxillectomy and right-sided neck dissection) identified a $3.5-\mathrm{cm}$ well-differentiated squamous cell carcinoma involving the alveolar ridge and maxillary sinus with bone invasion; level I and II (but not III/IV) nodes were involved. Perineural and lymphovascular invasion was noted. He underwent adjuvant radiation therapy with 6000 cGy over 6 wk, which included the adjacent lymph nodes. Initially, he made a good recovery, but unfortunately after 3 mo of follow-up he presented with a 4-cm neck mass and a fine needle aspirate-confirmed cancer recurrence. The mass was considered surgically unresectable and was within the previous radiation field. Thus, he was referred for palliative systemic therapy.

At that time he was consented for participation in the Personalized Oncogenomics (POG) study at BC Cancer in Vancouver, British Columbia (see Methods), and a biopsy of his neck mass was taken for genomic and transcriptomic analysis as per study protocol. He was also enrolled on a clinical trial of avelumab plus an OX40 agonist as his first systemic therapy. After 3 mo of treatment, he had markedly progressed (Fig. 1), and this treatment was discontinued. Based on the findings of the POG analysis, treatment with weekly $5-\mathrm{FU}\left(500 \mathrm{mg} / \mathrm{m}^{2}\right)$ and leucovorin $\left(20 \mathrm{mg} / \mathrm{m}^{2}\right)$ was initiated. He had a rapid response (Fig. 1) both clinically and radiographically that was sustained for $17 \mathrm{wk}$. He did require a dose modification because of side effects (specifically mucositis and hand-foot syndrome) and was maintained on $80 \%$ dose receiving a total of $14 \mathrm{wk}$ of treatment. A treatment break was initiated, but $3 \mathrm{wk}$ following treatment cessation, a small cancer nodule reappeared. A repeat biopsy was taken for POG sequencing and analysis that did not reveal marked changes in the genome or 

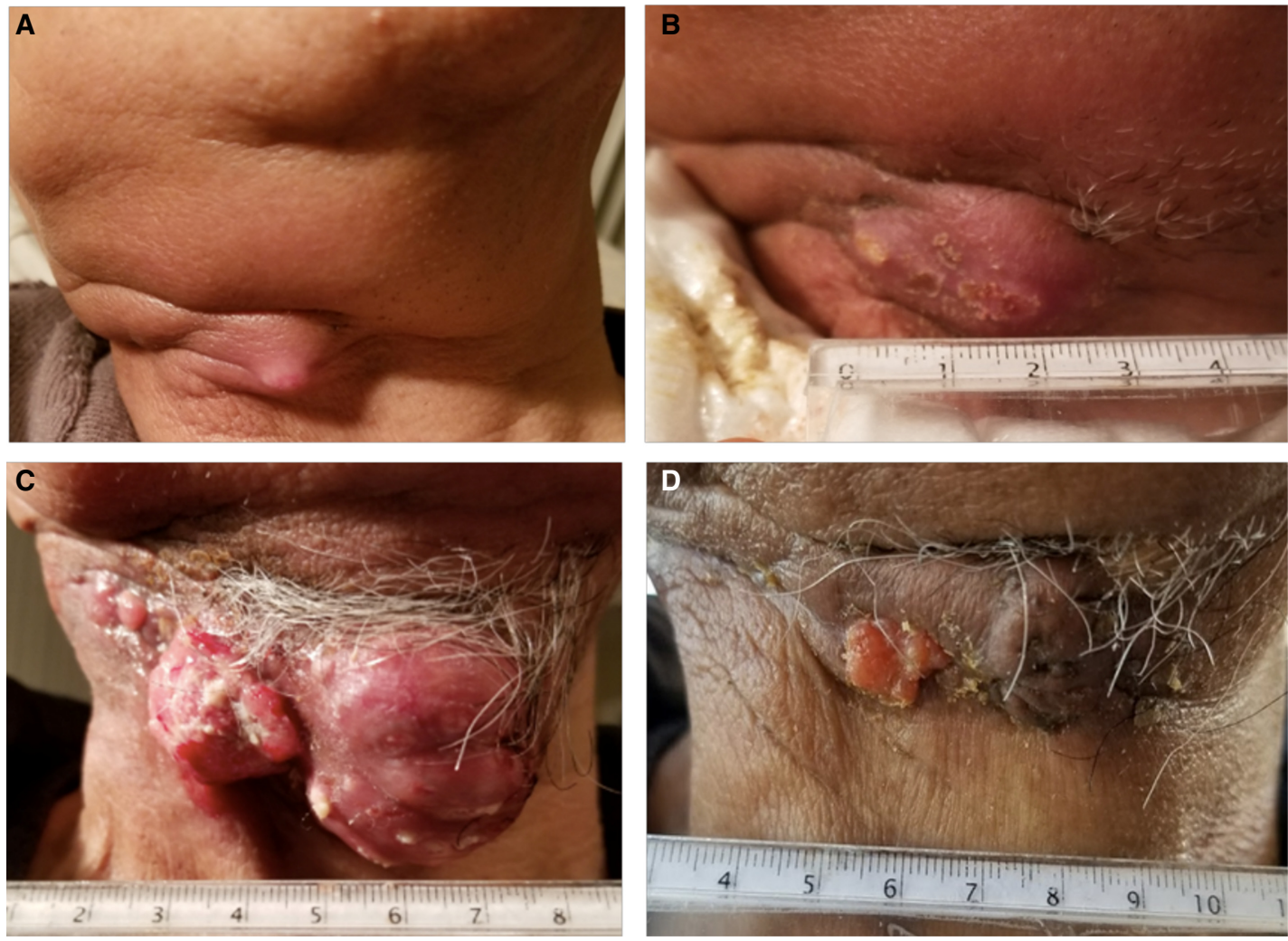

Figure 1. Clinical images of the metastatic deposits of this squamous cell carcinoma, originally surgically resected from the alveolar ridge. Images are organized chronologically starting from the baseline image $(A)$ when the metastatic nodule presented clinically and the biopsy for the POG analysis was taken. Following this, $B$ demonstrates the interim growth and representing the baseline image for the initiation of the clinical trial protocol of avelumab plus an OX40 agonist. $C$ depicts the growth of the cancer despite this systemic therapy. Finally, $D$ demonstrates the marked improvement after only 4 wk of weekly 5-FU/folinic acid therapy.

transcriptome compared with the initial biopsy. The patient was started on capecitabine (oral $5-\mathrm{FU})$, but unfortunately he did not respond to the reinitiation of this line of therapy. The mechanisms underlying the acquired resistance remain unclear. He was subsequently treated with cisplatin (progression as best response) and palbociclib (mixed response) on a clinical trial; unfortunately, he passed away because of tumor rupture and massive bleeding after 9 wk on trial.

\section{Genomic Analysis}

HPV genetic material was not detected in this tumor, further confirming the HPV-negative status identified in pathology. All relevant mutations and variants identified in the initial biopsy were confirmed in both the initial and repeat biopsies.

\section{Single-Nucleotide Variants}

We identified 85 somatic nonsynonymous protein-coding single-nucleotide variants (SNVs) from the sequencing data (Supplemental Table 1). Although none of these were deemed to be clinically actionable, three were of biological interest (Table 1). A homozygous promoter mutation (g.1295228G >A) at a recurrent hotspot was detected in the human telomerase reverse transcriptase (TERT) gene, an enzyme that maintains telomere length and genomic 
Table 1. Somatic nonsynonymous single-nucleotide variant (SNV) and indels

\begin{tabular}{lrrllllllll}
\hline Gene & Chr & Position & Ref & Alt & Type & HGVS CDS & HGVS protein & Genotype & Predicted effect & dbSNP \\
\hline KEAP1 & 19 & 10602878 & G & A & SNV & c.700C>T & p.Arg234Trp & Heterozygous & Missense & - \\
SLC29A1 & 6 & 44195070 & C & T & SNV & c.20C>T & p.Pro7Leu & Homozygous & Missense & - \\
TERT & 5 & 1295228 & C & T & SNV & c. $-124 C>T$ & - & Homozygous & - & rs1242535815 \\
\hline
\end{tabular}

(HGVS CDS) Human Genome Variation Society coding sequence, (dbSNP) Single Nucleotide Polymorphism Database.

integrity. Routine comparative analysis of gene expression revealed the TERT gene was moderately overexpressed compared with the Cancer Genome Atlas (TCGA) HNSCC data set (78th percentile). TERT promoter mutations occur in patients with oral cavity squamous cell carcinoma (SCC) at a high frequency and may be associated with aggressive disease (Killela et al. 2013; Zhao et al. 2015; Barczak et al. 2017; Chang et al. 2017). Additionally, there was a heterozygous variant (p.R234W) in KEAP1, a gene that is recurrently inactivated and associated with reduced survival in HNSCC (Network CGA 2015). Finally, a novel homozygous variant (p.P7L) was identified in nucleoside transporter SLC29A1 (hENT1). The functional and clinical impact of this mutation has not been characterized. However, hENT1 is important in 5-FU transport, and low levels of mRNA expression have been associated with response to 5-FU in pancreatic cancer cell lines (Tsujie et al. 2007). In this case, the somatic variant was also associated with a low level of SLC29A1 expression (5th percentile compared with TCGA HNSCC).

\section{Copy-Number Variants}

Based on the tumor/normal sequencing ratio and loss of heterozygosity, the tumor content was estimated to be $78 \%$ and a triploid model was used to describe the observed copy-number changes. Of particular interest, the genome-wide copy-number analysis revealed a homozygous CDKN2A/CDKN2B/MTAP codeletion. CDKN2A ( 1616$)$ is a tumor suppressor that regulates the cell cycle and is the second most commonly inactivated gene in HNSCC (Beck and Golemis 2016). Disruption of p16 allows for activation of CDK4 and CDK6 and phosphorylation of RB1, leading to cell cycle progression (Asghar et al. 2015). MTAP is also thought to function as a tumor suppressor and is a key enzyme in the formation of adenine, influencing response to 5-FU therapy (Tang et al. 2012).

\section{DPYD Structural Variant}

Structural variants were identified using de novo sequence assembly followed by variant detection. A somatic fusion deletion event in the DPYD gene predicted to result in an inframe deletion of exons 11-19 was detected in the genome and transcriptome (Fig. 2). The expression of the DPYD gene was moderate compared with the TCGA HNSCC data set (35th percentile). To our knowledge, our case is the first description of a somatic multiexon deletion in DPYD in a HNSCC cancer patient.

Evaluation of normal DNA revealed that the patient did not harbor any of the three SNP alleles associated with 5-FU toxicity (rs55886062; rs67376798; rs3918290) nor evidence of germline deletion in DPYD.

\section{DISCUSSION}

Here, we describe a case study of a patient with HNSCC who demonstrated a notable response to 5-FU. Alterations affecting multiple genes that play a role in nucleotide transport and metabolism were identified in this case study and may have contributed to the response 

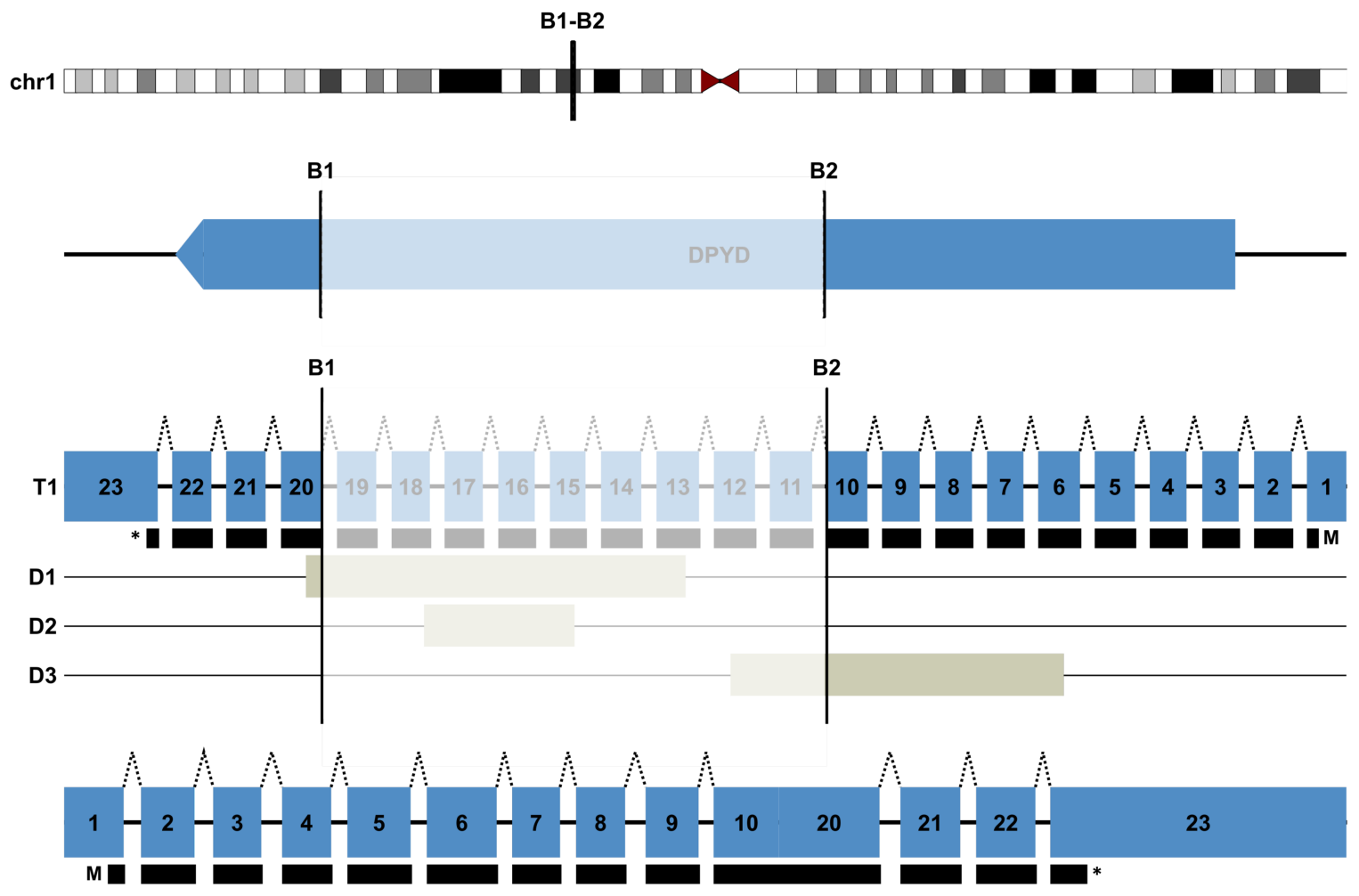

Figure 2. Somatic fusion deletion event in the DPYD gene. The plot depicts the breakpoints affecting the DPYD gene that was predicted to result in an in-frame deletion of exons 11-19. (Top) Deletion breakpoints B1 and B2 shown in relation to the chromosome. (Middle) Model of DPYD transcript ENST00000370192 (T1) depicting exons 1-23. DPYD contains three Pfam domains, which are depicted by D1 (PF01180: dihydro-orotate dehydrogenase), D2 (PF01207: TRNA-dihydrouridine synthase), and D3 (PF07992: Pyridine nucleotide-disulphide oxidoreductase). (Bottom) Resulting variant DPYD transcript lacking exons 11-19. Black blocks below the exons depict the protein-coding region of the transcript.

to therapy, including a novel somatic structural variant affecting DPYD. Discrete germline DPYD polymorphisms and deletions have been identified in cancer patients with severe 5-FU-associated toxicity (van Kuilenburg 2004; Etienne-Grimaldi et al. 2017; van Kuilenburg et al. 2017; Henricks et al. 2018). Thus, testing for DPYD variants known to affect DPD enzyme activity is becoming more prominent in patients undergoing 5-FU chemotherapy (Deenen et al. 2016). Our case was found to be negative for described germline alterations associated with 5-FU toxicity. However, the somatic structural variant analysis resulted in the identification of a rearrangement in the DPYD gene leading to a partial inframe deletion of exons 11-19. This region of the gene contains part of the FAD-binding domain and the majority of the FMN/pyrimidine binding domain (van Kuilenburg 2004), which likely results in the translation of a nonfunctional protein that is unable to metabolize 5-FU. We, therefore, speculated that the tumor may be sensitive to 5-FU/capecitabine because of the somatic DPYD structural variant. Indeed, this patient demonstrated a rapid and dramatic reduction in tumor size following initiation of treatment with 5-FU, which lasted for $17 \mathrm{wk}$. This notable response was of particular interest as his cancer was resistant to other standard treatments. In support of the hypothesis that the somatic DPYD loss may contribute to 5-FU response, another study that conducted a retrospective analysis of triple-negative breast cancer patients revealed patients with somatic DPYD copy-number variants (CNVs) demonstrated a trend for a longer time to progression on 5-FU (Gross et al. 2013). 
COLD SPRING HARBOR Molecular Case Studies
DPYD variant and 5-FU sensitivity in a HNSCC case
Unfortunately, the patient relapsed within 3 wk of ending 5-FU therapy and was subsequently reinitiated on oral 5-FU, capecitabine, but did not respond. Repeat biopsy prior to initiation of capecitabine did not reveal any further alterations of note, and the mechanism of resistance remains unclear.

Additional genomic findings that may also contribute to 5-FU response include a variant of unknown significance affecting SLC29A1 and homozygous deletion of MTAP. High expression of SLC29A1 has been associated with resistance to 5-FU in pancreatic cancer cell lines (Tsujie et al. 2007) by either facilitating bilateral transport of 5-FU or by preferential transport of nucleosides over 5-FU into cells (Wang et al. 2014). Although the low expression of SLC29A1 in the tumor has been associated with a clinical response to 5-FU (Tsujie et al. 2007; Phua et al. 2013), the homozygous single nucleotide variant (p.P7L) has not been observed in any public databases to date and is located on the amino terminus of the protein with no associated functional domain. Therefore, the precise clinical impact of this variant on 5-FU response remains unclear. Additionally, MTAP deletion is commonly found in a number of different cancer types (Tang et al. 2012) including oral SCCs (Chen et al. 2004). Adenine derived from MTAP activity could compete with purine analogs such as 5-FU for phosphoribosyl-5-pyrophosphate substrate utilization, thereby decreasing the amount of toxic nucleotide produced (Tang et al. 2012). Thus, the deletion of MTAP could result in increased amounts of toxic analog. In line with this hypothesis, several in vitro studies have shown that the deletion of MTAP in various cell lines resulted in the enhanced cytotoxicity in response to 5-FU treatment (Lubin and Lubin 2009; Lubin and Lubin 2010; Tang et al. 2012). However, there is no clinical evidence of its impact on 5-FU treatment to date. Altogether, the genomic findings point to the inability of the tumor to catabolize and deactivate 5-FU and therefore increase its therapeutic efficacy.

Structural variants and inactivating mutations in DPYD are rare events, with only nine cases cataloged in TCGA and COSMIC databases (cBioportal; Cerami et al. 2012; Gao et al. 2013). DPYD alterations were found in a variety of different cancer types, highlighting the potential impact that somatic mutations can have on treatment choices and outcomes. By sequencing the whole genome, we were positioned to find novel variants, including the DPYD structural variant described here, that informed personalized patient management that may otherwise be missed in targeted sequencing approaches.

\section{CONCLUSION}

Comprehensive genomic and transcriptomic analysis of a metastatic HNSCC was performed. A somatic structural variant affecting DPYD was detected that lead to the patient receiving 5-FU therapy. The patient had a rapidly growing cancer that was resistant to radiation, immunotherapy, and cisplatin and yet he demonstrated an impressive clinical response to 5-FU therapy, which we hypothesize to be in part due to the DPYD structural variant. This analysis shows for the first time that somatic lesions in DPYD may be used as a potential biomarker in prospectively evaluating for somatic variants that point to treatment sensitivity in patients with HNSCC and other cancer types in which 5-FU is commonly used.

\section{METHODS}

\section{Sample Collection and Processing}

The patient was enrolled in the ongoing POG project at BC Cancer in Vancouver, Canada (NCT02155621). This study was approved by the University of British Columbia Research Ethics Board (REB\#H14-006817). Patient identity is de-identified for the research team 
Table 2. Sequencing coverage

\begin{tabular}{lccc}
\hline Sample & $\begin{array}{c}\text { Tumor DNA coverage } \\
\text { (WGS) }\end{array}$ & $\begin{array}{c}\text { Normal DNA coverage } \\
\text { (WGS) }\end{array}$ & $\begin{array}{c}\text { Tumor RNA coverage } \\
\text { (RNA-seq) }\end{array}$ \\
\hline HNSCC case & $101 \times$ & $39 \times$ & $360 \mathrm{M}$ reads \\
\hline
\end{tabular}

and information is communicated to the clinicians through unique patient identifiers. Patients consent to the potential publication of findings. Raw sequence data, analytics, and clinical data are maintained in secure computing environments.

As per study protocol, each patient undergoes a study-specific biopsy. In this case, an ultrasound-guided core-needle biopsy of the neck and blood samples were collected for paired-end whole-genome sequencing (WGS) and transcriptome sequencing. RNA and DNA libraries were prepared and sequenced on the Illumina platform as previously described (Grewal et al. 2017).

\section{Sequencing Data Analysis}

In total, 150 bp paired-end normal and tumor reads were aligned to the human reference genome (hg19) using the Burrows-Wheeler alignment tool (Li and Durbin 2010) (v0.7.6a). Somatic point mutations and small insertion and deletions were detected using Strelka (v1.0.6) (Saunders et al. 2012). Somatic copy-number alterations that were present in the tumor DNA but not in the germline were identified using CNAseq (v0.0.6) (Jones et al. 2010) and loss of heterozygosity was determined using APOLLOH (v0.1.1) (Ha et al. 2012). De novo assembly of genomic and transcriptomic data using ABySS v1.3.4 (Simpson et al. 2009) and TransABySS (v1.4.10) (Birol et al. 2009; Simpson et al. 2009) was carried out to detect rearrangements. Mutations were annotated to Ensembl v69 (Flicek et al. 2014) using SNPEff (v4.1) (Cingolani et al. 2012). Tumor content and sequencing coverage are outlined in Table 2.

RNA-seq reads were aligned using Jaguar (v2.0.3) (Butterfield et al. 2014) to the human reference (hg19) with a database of exon junctions based on Ensembl v69 (Flicek et al. 2014), and normalized expression levels were computed in reads per kilobase per million mapped reads (Mortazavi et al. 2008). Publicly available transcriptome sequencing data from HNSCC from TCGA (https://tcga-data.nci.nih.gov/tcga/; Network CGA 2015) and a compendium of adjacent normal tissue samples from the Illumina Human BodyMap 2.0 project (www .illumina.com; ArrayExpress ID: E-MTAB-513) were used to explore the expression profile of human genes and transcripts.

\section{ADDITIONAL INFORMATION}

\section{Database Deposition and Access}

The whole-genome and transcriptome sequencing data for this case are available as .bam files from the European Genome-phenome Archive (EGA; www.ebi.ac.uk/ega/home) as part of the study EGAS00001001159, accession ID EGAD00001004934. The DPYD structural variant and variants affecting KEAP1 and SLC29A1 are deposited in ClinVar (https: www.ncbi.nlm.nih.gov/clinvar/) under accession numbers SCV000996023, SCV000996 021, and SCV000996022, respectively.

\section{Ethics Statement}

The patient provided written informed consent for metastatic biopsies, sequencing, and publication of results as part of the Personalized Oncogenomics Program of British Columbia (NCT02155621, University of British Columbia Clinical Research Ethics Board approval no. H14-006817). 
COLD SPRING HARBOR Molecular Case Studies
DPYD variant and 5-FU sensitivity in a HNSCC case
Competing Interest Statement

The authors have declared no competing interest.

Received August 16, 2019; accepted in revised form November 18, 2019

\section{Acknowledgments}

This work would not be possible without the participation of our patients and families, the POG team, and the generous support of the BC Cancer Foundation and Genome British Columbia (project B20POG). We also acknowledge contributions toward equipment and infrastructure from Genome Canada and Genome BC (projects 202SEQ, 212SEQ, 12002), Canada Foundation for Innovation (projects 20070, 30981, 30198, 33408), and the BC Knowledge Development Fund. The results published here are in part based on data generated by the following projects and obtained from dbGaP (http://www.ncbi.nlm.nih.gov/gap): The Cancer Genome Atlas managed by the National Cancer Institute ( $\mathrm{NCl}$ ) and the National Human Genome Research Institute (NHGRI) (http://cancergenome.nih.gov) and the Genotype-Tissue Expression (GTEx) Project, supported by the Common Fund of the Office of the Director of the National Institutes of Health (https://commonfund.nih.gov/GTEx).

\section{REFERENCES}

Adelstein D, Gillison ML, Pfister DG, Spencer S, Adkins D, Brizel DM, Burtness B, Busse PM, Caudell JJ, Cmelak AJ, et al. 2017. NCCN guidelines insights: head and neck cancers, Version 2.2017. J Natl Compr Canc Netw 15: 761-770. doi:10.6004/jnccn.2017.0101

Asghar U, Witkiewicz AK, Turner NC, Knudsen ES. 2015. The history and future of targeting cyclin-dependent kinases in cancer therapy. Nat Rev Drug Discov 14: 130-146. doi:10.1038/nrd4504

Barczak W, Suchorska WM, Sobecka A, Bednarowicz K, Machczynski P, Golusinski P, Rubis B, Masternak MM, Golusinski W. 2017. hTERT C250T promoter mutation and telomere length as a molecular markers of cancer progression in patients with head and neck cancer. Mol Med Rep 16: 441-446. doi:10.3892/mmr.2017 .6590

Beck TN, Golemis EA. 2016. Genomic insights into head and neck cancer. Cancers Head Neck 1: 10. doi:10 $.1186 / s 41199-016-0012-y$

Berman TA, Schiller JT. 2017. Human papillomavirus in cervical cancer and oropharyngeal cancer: one cause, two diseases. Cancer 123: 2219-2229. doi:10.1002/cncr.30588

Birol I, Jackman SD, Nielsen CB, Qian JQ, Varhol R, Stazyk G, Morin RD, Zhao Y, Hirst M, Schein JE, et al. 2009. De novo transcriptome assembly with ABySS. Bioinformatics 25: 2872-2877. doi:10.1093/bioinformatics/ btp367

Butterfield YS, Kreitzman M, Thiessen N, Corbett RD, Li Y, Pang J, Ma YP, Jones SJ, Birol I. 2014. JAGuaR: junction alignments to genome for RNA-seq reads. PLoS One 9: e102398. doi:10.1371/journal.pone .0102398

Cerami E, Gao J, Dogrusoz U, Gross BE, Sumer SO, Aksoy BA, Jacobsen A, Byrne CJ, Heuer ML, Larsson E, et al. 2012. The cBio cancer genomics portal: an open platform for exploring multidimensional cancer genomics data. Cancer Discov 2: 401-404. doi:10.1158/2159-8290.CD-12-0095

Chang K-P, Wang C-I, Pickering CR, Huang Y, Tsai C-N, Tsang N-M, Kao H-K, Cheng M-H, Myers JN. 2017. Prevalence of promoter mutations in the TERT gene in oral cavity squamous cell carcinoma. Head Neck 39: 1131-1137. doi:10.1002/hed.24728

Chen Y-J, Lin S-C, Kao T, Chang C-S, Hong P-S, Shieh T-M, Chang K-W. 2004. Genome-wide profiling of oral squamous cell carcinoma. J Pathol 204: 326-332. doi:10.1002/path.1640

Cingolani P, Platts A, Wang le L, Coon M, Nguyen T, Wang L, Land SJ, Lu X, Ruden DM. 2012. A program for annotating and predicting the effects of single nucleotide polymorphisms, SnpEff: sNPs in the genome of Drosophila melanogaster strain $\mathrm{w}^{1118}$; iso-2; iso-3. Fly (Austin) 6: 80-92. doi:10.4161/fly.19695

Deenen MJ, Meulendijks D, Cats A, Sechterberger MK, Severens JL, Boot H, Smits PH, Rosing H, Mandigers CM, Soesan M, et al. 2016. Upfront genotyping of DPYD*2A to individualize fluoropyrimidine therapy: a safety and cost analysis. J Clin Oncol 34: 227-234. doi:10.1200/JCO.2015.63.1325

Diasio RB, Harris BE. 1989. Clinical pharmacology of 5-fluorouracil. Clin Pharmacokinet 16: 215-237. doi:10 .2165/00003088-198916040-00002

Etienne-Grimaldi M-C, Boyer J-C, Beroud C, Mbatchi L, van Kuilenburg A, Bobin-Dubigeon C, Thomas F, Chatelut E, Merlin J-L, Pinguet F, et al. 2017. New advances in DPYD genotype and risk of severe toxicity under capecitabine. PLoS One 12: e0175998. doi:10.1371/journal.pone.0175998

Flicek P, Amode MR, Barrell D, Beal K, Billis K, Brent S, Carvalho-Silva D, Clapham P, Coates G, Fitzgerald S, et al. 2014. Ensembl 2014. Nucleic Acids Res 42: D749-D755. doi:10.1093/nar/gkt1196

Fung N, Faraji F, Kang H, Fakhry C. 2017. The role of human papillomavirus on the prognosis and treatment of oropharyngeal carcinoma. Cancer Metastasis Rev 36: 449-461. doi:10.1007/s10555-017-9686-9 
Gao J, Aksoy BA, Dogrusoz U, Dresdner G, Gross B, Sumer SO, Sun Y, Jacobsen A, Sinha R, Larsson E, et al. 2013. Integrative analysis of complex cancer genomics and clinical profiles using the cBioPortal. Sci Signal 6: pl1. doi:10.1126/scisignal.2004088

Grewal JK, Eirew P, Jones M, Chiu K, Tessier-Cloutier B, Karnezis AN, Karsan A, Mungall A, Zhou C, Yip S, et al. 2017. Detection and genomic characterization of a mammary-like adenocarcinoma. Cold Spring Harb Mol Case Stud 3: a002170. doi:10.1101/mcs.a002170

Gross E, Meul C, Raab S, Propping C, Avril S, Aubele M, Gkazepis A, Schuster T, Grebenchtchikov N, Schmitt $M$, et al. 2013. Somatic copy number changes in DPYD are associated with lower risk of recurrence in triplenegative breast cancers. Br J Cancer 109: 2347-2355. doi:10.1038/bjc.2013.621

Ha G, Roth A, Lai D, Bashashati A, Ding J, Goya R, Giuliany R, Rosner J, Oloumi A, Shumansky K, et al. 2012. Integrative analysis of genome-wide loss of heterozygosity and monoallelic expression at nucleotide resolution reveals disrupted pathways in triple-negative breast cancer. Genome Res 22: 1995-2007. doi:10 $.1101 /$ gr.137570.112

Henricks LM, Siemerink EJM, Rosing H, Meijer J, Goorden SMI, Polstra AM, Zoetekouw L, Cats A, Schellens JHM, van Kuilenburg ABP. 2018. Capecitabine-based treatment of a patient with a novel DPYD genotype and complete dihydropyrimidine dehydrogenase deficiency. Int J Cancer 142: 424-430. doi:10.1002/ijc .31065

Jones SJ, Laskin J, Li YY, Griffith OL, An J, Bilenky M, Butterfield YS, Cezard T, Chuah E, Corbett R, et al. 2010. Evolution of an adenocarcinoma in response to selection by targeted kinase inhibitiors. Genome Biol 11: R82. doi:10.1186/gb-2010-11-8-r82

Killela PJ, Reitman ZJ, Jiao Y, Bettegowda C, Agrawal N, Diaz LA, Friedman AH, Friedman H, Gallia GL, Giovanella BC, et al. 2013. TERT promoter mutations occur frequently in gliomas and a subset of tumours derived from cells with low rates of self-renewal. Proc Natl Acad Sci USA 110: 6021-6026. doi:10.1073/ pnas. 1303607110

Li H, Durbin R. 2010. Fast and accurate long-read alignment with Burrows-Wheeler transform. Bioinformatics 26: 589-595. doi:10.1093/bioinformatics/btp698

Lubin M, Lubin A. 2009. Selective killing of tumours deficient in methylthioadenosine phosphorylase: a novel strategy. PLoS One 4: e5735. doi:10.1371/journal.pone.0005735

Lubin M, Lubin A. 2010. Correction: selective killing of tumours deficient in methylthioadenosine phosphorylase: a novel strategy. PLoS One 4: e5735. doi:10.1371/annotation/54fad81d-c975-4b30-bb2b-249650ec3d66

Meulendijks D, Henricks LM, Sonke GS, Deenen MJ, Froehlich TK, Amstutz U, Largiader CR, Jennings BA, Marinaki AM, Sanderson JD, et al. 2015. Clinical relevance of DPYD variants c.1679T>G, c.1236G>A/ HapB3, and c.1601G>A as predictors of severe fluoropyrimidine-associated toxicity: a systematic review and meta-analysis of individual patient data. Lancet Oncol 16: 1639-1650. doi:10.1016/S1470-2045(15) 00286-7

Mortazavi A, Williams BA, McCue K, Schaeffer L, Wold B. 2008. Mapping and quantifying mammalian transcriptomes by RNA-Seq. Nat Methods 5: 621-628. doi:10.1038/nmeth.1226

Network CGA. 2015. Comprehensive genomic characterization of head and neck squamous cell carcinomas. Nature 517: 576-582. doi:10.1038/nature14129

Phua LC, Mal M, Koh PK, Cheah PY, Chan EC, Ho HK. 2013. Investigating the role of nucleoside transporters in the resistance of colorectal cancer to 5-fluorouracil therapy. Cancer Chemother Pharmacol 71: 817-823. doi:10.1007/s00280-012-2054-0

Saunders CT, Wong WS, Swamy S, Becq J, Murray LJ, Cheetham RK. 2012. Strelka: accurate somatic smallvariant calling from sequenced tumor-normal sample pairs. Bioinformatics 28: 1811-1817. doi:10.1093/ bioinformatics/bts271

Simpson JT, Wong K, Jackman SD, Schein JE, Jones SJ, Birol I. 2009. ABySS: a parallel assembler for short read sequence data. Genome Res 19: 1117-1123. doi:10.1101/gr.089532.108

Tang B, Testa JR, Kruger WD. 2012. Increasing the therapeutic index of 5-fluorouracil and 6-thioguanine by targeting loss of MTAP in tumour cells. Cancer Biol Ther 13: 1082-1090. doi:10.4161/cbt.21115

Tsujie M, Nakamori S, Nakahira S, Takahashi Y, Hayashi N, Okami J, Nagano H, Dono K, Umeshita K, Sakon M, et al. 2007. Human equilibrative nucleoside transporter 1, as a predictor of 5-fluorouracil resistance in human pancreatic cancer. Anticancer Res 27: 2241-2249. PMID:17695509

van Kuilenburg AB. 2004. Dihydropyrimidine dehydrogenase and the efficacy and toxicity of 5-fluorouracil. Cancer 40: 939-950. doi:10.1016/j.ejca.2003.12.004

van Kuilenburg AB, Meijer J, Maurer D, Dobritzsch D, Meinsma R, Los M, Knegt LC, Zoetekouw L, Jansen RL, Dezentje V, et al. 2017. Severe fluoropyrimidine toxicity due to novel and rare DPYD missense mutations, deletion and genomic amplification affecting DPD activity and mRNA splicing. Biochim Biophys Acta Mol Basis Dis 1863: 721-730. doi:10.1016/j.bbadis.2016.12.010

Wang WB, Yang Y, Zhao YP, Zhang TP, Liao Q, Shu H. 2014. Recent studies of 5-fluorouracil resistance in pancreatic cancer. World J Gastroenterol 20: 15682-15690. doi:10.3748/wjg.v20.i42.15682

Zhao T, Hu F, Qiao B, Chen Z, Tao Q. 2015. Telomerase reverse transcriptase potentially promotes the progression of oral squamous cell carcinoma through induction of epithelial-mesenchymal transition. Int $J$ Oncol 46: 2205-2215. doi:10.3892/ijo.2015.2927 


\section{COLD SPRING HARBOR Molecular Case Studies}

\section{Fluorouracil sensitivity in a head and neck squamous cell carcinoma with a somatic DPYD structural variant}

Elisa Majounie, Kathleen Wee, Laura M. Williamson, et al.

Cold Spring Harb Mol Case Stud 2020, 6: a004713 originally published online December 23, 2019 Access the most recent version at doi: $10.1101 /$ mcs.a004713
Supplementary http://molecularcasestudies.cshlp.org/content/suppl/2020/01/09/mcs.a004713.D Material C1
References This article cites 38 articles, 9 of which can be accessed free at: http://molecularcasestudies.cshlp.org/content/6/1/a004713.full.html\#ref-list-1
License This article is distributed under the terms of the Creative Commons Attribution-NonCommercial License, which permits reuse and redistribution, except for commercial purposes, provided that the original author and source are credited.
Email Alerting Receive free email alerts when new articles cite this article - sign up in the box at the Service top right corner of the article or click here.

